\title{
Essentials of cell physiology
}

Dr Maike Glitsch is Associate Professor in Biomedical Sciences in the Department of Physiology, Anatomy and Genetics, University of Oxford, UK.

\begin{abstract}
Cell physiology investigates how cells function and interact with their environment. Cells are exposed to a range of distinct stimuli in response to which they need to initiate appropriate responses to ensure their own survival as well as that as that of the organism as a whole. Responding to stimuli is only possible if cells express receptors capable of sensing the presence of these stimuli. An important role in this process is played by receptors that are inserted in the membrane surrounding and protecting cells, as they permit cells to sense stimuli without directly exposing their intracellular milieu to them. Receptors can detect a number of distinct stimuli, chemical, mechanical and thermal, and electrical. The response triggered in a cell following receptor stimulation depends on the type of receptor and downstream signalling molecules present in the cell as well as the presence or absence of other stimuli. A good understanding of cell physiology is of high importance to surgeons as it helps understand, predict and improve clinical outcome and devise treatment strategies.
\end{abstract}

Key words: cell membrane; ion channels; G protein coupled receptors; transporters; enzyme-linked kinases, microenvironment; signalling; second messengers

\section{Introduction}

Cell physiology is concerned with how cells, the building blocks of tissues and organs, function, and how they interact with each other and their environment. In complex, multicellular organisms such as mammals, an interaction between cells and their surroundings is essential for the survival of the organism. All mammalian cells have the same general organisation and organelles (see Text Box 1 for a quick guide to organelles and their functions), yet different cells within the body have to fulfil distinct roles. This is achieved through differences in cell shape including specialisations such as dendrites or cilia, and cell-specific expression of proteins that convey distinct properties to 
the cells that express them. Of particular importance here are proteins embedded in the membrane, as they represent a link between extra- and intracellular environment and determine how cells respond to external stimuli.

\section{Microenvironment}

Cells do not exist and function in isolation but they are surrounded by other cells, which they may be physically connected to (in tissues) or not (e.g. immune cells). They are also surrounded by interstitial fluid, which contains ions and other chemicals. The sum of all the cells and chemicals that surround a cell as well as prevailing physical factors, such as temperature, impacting on cells is called the microenvironment of that cell. Crucially, cells can sense changes in their microenvironment, whether these changes are chemical, mechanical, electrical or thermal. This ability to detect changes in their environment is the fundamental basis that allows cells to respond appropriately to externally generated stimuli, such as starting or stopping to proliferate or releasing chemicals, and to communicate with other cells.

Cells in different tissues have different cellular neighbours, and the interstitial fluids of distinct tissues differ in their composition. Importantly, the interstitial fluid composition can change in a cell and tissue activity dependent manner. Hence, cells alter and shape their own microenvironment and that of neighbouring cells. Whether or not changes in the cellular microenvironment are picked up by a cell depends on the presence of sensors, or receptors, for the parameter that has changed, and on the ability of the stimulus to reach that receptor.

\section{Cell Membrane}

All eukaryotic cells are surrounded by a cell, or plasma, membrane, a lipid bilayer made up of hundreds to thousands of distinct lipids. It acts as a diffusion barrier that controls which chemicals can cross into and out of cells. Very small and hydrophobic chemicals as well as gases can diffuse through the plasma membrane unaided. All other chemicals, including ions, need to be transported across with the help of specialised proteins that are inserted in the membrane, channel and carrier proteins. This selective permeability of membranes to chemicals and in particular to ions results in the generation of a 
membrane potential across the membrane that, in turn, influences transport processes and allows cells to create and respond to electrical signals.

Not all chemical or other stimuli can or need to cross the membrane to have an effect. The plasma membrane offers a surface for insertion of sensors/receptors that can detect changes in virtually all aspects of the cellular microenvironment. Intriguingly, distribution of receptors across the membrane is not uniform. The membrane presents a scaffold to enable creation of niches of specialised activity. This is achieved via lipid rafts: specialised membrane microdomains that enable cells to compartmentalise cellular processes by serving as organising centres for specific signalling events. Lipid rafts consist of membrane lipids and receptor proteins and may differ in structure and function depending on their composition.

This non-uniformity is not limited to proteins but also applies to the lipids that make up the membrane, and stimulus-driven hydrolysis of membrane lipids can lead to the generation of extra- and intracellular signalling molecules.

\section{Cell membrane receptors}

Activation of cell surface receptors by chemical, mechanical, electrical or thermal stimuli invariably results in conformational changes of the receptor protein, which in turn cause knock-on effects intracellularly and affect intracellular signalling cascades, culminating in altered intracellular processes. In this way, extracellular changes are transduced intracellularly. Importantly, a cell is unaware of changes in its environment if it does not express the appropriate receptors that allow it to sense these changes. The different stimulus modalities (chemical, mechanical, electrical, thermal) are sensed by receptors that specialise in picking up these stimuli.

Chemical stimuli directly bind to their receptor and include a range of distinct compounds, such as break-down products of complex molecules that indicate cell damage or pathogen invasion, or hormones. Significantly, receptors may also sense the absence of molecules that would normally be present, in which case the unbinding of a usually present chemical and subsequent termination of a normally ongoing signalling cascade is meaningful to the cell.

Mechanical stimuli include changes in pressure on the cell membrane, which can be brought about through cell shrinking or swelling; these processes are involved in osmosensing and are crucial for body water homeostasis. Moreover, cells may sense pressure being applied to them either directly or due 
to deformation of neighbouring cells (e.g. contraction of muscle cells). Receptors sensing pressure changes are referred to as mechanosensitive or stretch-activated, and their exact mode of action is still unclear.

Thermal changes in the microenvironment can arise due to high respiratory activity of cells (e.g. skeletal muscle cells, immune cells). Receptor proteins are rendered thermosensitive by exhibiting exquisite temperaturedependence of distinct conformational states. Hence, cold temperatures may energetically favour an inactive receptor form whereas warm temperatures may facilitate transition to the activated receptor, or vice versa.

Changes in the electrical environment occur when the ion and hence charge composition of the extra- and/or intracellular fluid changes. Unlike chemical receptors, voltage-sensing receptors do not bind their stimulus (i.e. ions) directly but contain a sequence of charged amino acids that move in response to changes in their charge environment, thereby causing receptor activation.

It is important to appreciate that many receptors can multitask and sense the presence (or absence) of more than one stimulus. Different stimuli, if they don't serve as activators, may potentiate or inhibit receptor function. A prominent example for this is the effect of $\mathrm{pH}$, a measure of proton concentration, on protein function. Changes in $\mathrm{pH}$ reflect changes in a chemical stimulus that may activate or potentiate some, and inhibit other receptors. Equally, changes in membrane potential affect all transmembrane proteins, not just those specialising in picking up voltage changes. The extent of impact of membrane voltage depends on the amino acid composition and structure of the protein. Hence, receptor proteins may act as coincidence detectors that function differently depending on the combination of stimuli present.

Stimulated cells can communicate to other cells that they have received a stimulus. They may release chemicals in response to their stimulation (e.g. neurotransmitters, hormones), and these chemicals are then picked up by other cells through binding to their cell surface receptors. Released chemicals may also act as autocrine signals, by binding to receptors on the cells from which they were released. This may serve as a feed-back mechanism and can also provide a memory of past activity. Moreover, cellular stimulation may result in changes of the ionic composition of the interstitial fluid through opening or closing of ion channels, and this may affect the membrane potential of not only 
the stimulated, but also neighbouring cells. The same is true for increased metabolic activity, which may result in increases in ambient temperature (e.g. in inflamed tissue). Finally, mechanical stress or deformation of one cell can be sensed by neighbouring cells due to the fact that in tissues, cells are connected to each other via adhesive molecules including integrins and claudins.

Once receptors have been activated, mechanisms must be in place to switch them off again. One obvious way for terminating a response is the removal of the stimulus (for instance break-down or diffusion away of the chemical). Moreover, receptors may desensitise (or inactivate) in the continued presence of the stimulus, meaning that prolonged exposure to their stimulus causes a conformational change in the protein that renders it insensitive to the presence of the stimulus and hence switches off the receptor. Finally, receptors can be removed from the membrane by internalisation.

Receptor proteins fall into three main families, ion channel linked, enzyme linked, and $\mathrm{G}$ protein coupled receptors, and most stimuli activate more than one of these three families.

\section{Ion channel linked receptors (Ion channels)}

Ion channels are receptor proteins that contain a channel pore through which ions can leave or enter a cell. Aberrant ion channel activity is observed in almost all pathologies, and in channelopathies, channel dysfunction due to mutations in the channel gene is the primary cause of the disease. Examples of channelopathies include cystic fibrosis, episodic ataxia, short and long QT syndrome, and neonatal diabetes mellitus. Clinicians take advantage of pharmacological tools that affect the activity of certain channels, e.g. anaesthetic drugs (lidocaine inhibits voltage-gated $\mathrm{Na}^{+}$channels), muscle relaxants, and drugs that favourably alter mental states (e.g. benzodiazepines).

Channel opening and/or closing may be extremely fast; some channels respond to a stimulus within ms, whereas others take much longer to open. This is also true for closing of channels, which can be extremely rapid or take seconds or even minutes. Once a channel pore is open, ions diffuse through it across the membrane. The direction of ion flow is determined by the concentrations of the permeant ion on either side of the membrane (its concentration gradient), and the voltage across the membrane (the electric 
gradient); the movement of ions is affected by electrical potentials because ions are charge carriers. The combination of the electric and chemical gradients is called the electrochemical driving force for that particular ion.

Ion channels can be highly selective for a particular ion, the most selective ones displaying a selectivity of 99.999\% (store-operated $\mathrm{Ca}^{2+}$ channels) and even $100 \%$ (voltage-gated $\mathrm{H}^{+}$channels) for a given ion. However, not all channels show this high selectivity. Some are selective for cations over anions but do not distinguish well between cations, exhibiting similar permeabilities for $\mathrm{Na}^{+}$and $\mathrm{K}^{+}$, with some also being permeable to $\mathrm{Ca}^{2+}$ and even $\mathrm{Mg}^{2+}$. Anion channels tend to be permeable to both $\mathrm{Cl}^{-}$and bicarbonate, but different anion channel proteins may show preference for one over the other. It is important to appreciate that ion channels may exert effects beyond changes in membrane potential, and this is particularly true for ion channels that influence the basal intracellular $\mathrm{Ca}^{2+}$ concentration, which is generally very low in unstimulated cells (over 10,000 times lower than extracellular $\mathrm{Ca}^{2+}$ concentration). $\mathrm{Ca}^{2+}$ is a ubiquitous and powerful intracellular messenger that, when its concentration is increased, can influence cell fate in the long term by changing gene transcription events, but also affect cell motility, contraction state, and release of chemicals (e.g. neurotransmitter) from cells in the short term.

Many channels are selective for the stimulus that opens them but it is becoming increasingly clear that a number of ion channels can be activated by more than one stimulus. The TRPV1 channel protein, for example, is responsible for painful and hot sensations, and it is activated by the chemicals capsaicin (active ingredient in hot chilli peppers), protons (acid sensing), tarantula toxin (signalling pain), by (noxious) heat, membrane stretch (a truncated version of the channel protein), and changes in voltage across the membrane.

\section{Enzyme linked receptors}

These receptors either exhibit intracellular enzyme activity themselves or directly associate with intracellular enzymes that become active upon receptor stimulation following binding of their activating chemical. Most enzyme linked receptors display kinases activity, meaning that they phosphorylate target proteins, but there are also phosphatases (which dephosphorylate proteins) and 
guanylate cyclase receptors. The latter catalyse the formation of cyclic GMP (cGMP), a second messenger that can bind to and stimulate target proteins such as ion channels (cyclic nucleotide gated channels) or kinases (Protein Kinase $\mathrm{G})$.

Kinases fall into two categories depending on the amino acids that they phosphorylate in their target protein: tyrosine and serine/threonine kinases. The majority of receptor-linked kinases are tyrosine kinases, most of which are receptors for growth factors and hormones and promote cell division and growth. Unsurprisingly, maladaptive receptor tyrosine kinase activity has been linked to numerous cancers. Receptor Serine/Threonine kinases can equally influence gene transcription processes and affect cell fate.

Different kinases recognise distinct consensus sequences within which they phosphorylate their target amino acid. Hence, a protein may be subject to phosphorylation by several kinases and at separate sites, and each phosphorylation event has different consequences for the function of the protein. Phosphorylation of AMPA receptor ion channels at two separate sites, for instance, has opposite effects on the channel protein, with phosphorylation of one site causing its insertion into the membrane, while phosphorylation of another site causes it to be internalised.

\section{G protein coupled receptors (GPCRs)}

GPCRs, also called heptahelical, 7TM, or metabotropic receptors, can be grouped into six families based on sequence homology and function; four of these families are present in mammals. All GPCRs share the same basic structure of seven transmembrane segments, an extracellular ligand and intracellular $\mathrm{G}$ protein binding sites.

$G$ proteins are made up of three polypeptides $(\alpha, \beta$, and $\gamma)$; they dissociate from the receptor upon stimulation and split into two functional subunits, $\alpha$ and $\S \gamma$, which in turn interact with and thereby modify the activity of enzymes and ion channels. There are a large number of distinct $G$ proteins that link to different signalling cascades. The two most common $G$ protein effects are (1) changes in cyclic nucleotide levels (cAMP and cGMP), through activation or inhibition of adenylate cyclases (formation of cAMP) or phosphodiesterases (break-down of cAMP and cGMP), and (2) changes in intracellular $\mathrm{Ca}^{2+}$ concentration through mobilisation of $\mathrm{Ca}^{2+}$ from intracellular $\mathrm{Ca}^{2+}$ stores and 
activation of $\mathrm{Ca}^{2+}$ influx through plasma membrane ion channels. Cyclic nucleotides and intracellular $\mathrm{Ca}^{2+}$ in turn interact with enzymes, transcription factors, or ion channels, thereby profoundly influencing cell fate. In this way, complex and partially overlapping signalling cascades can follow GPCR activation, though this is not necessarily always the case.

Which signalling cascade is activated in response to GPCR activation depends on which $G$ proteins, enzymes and other signalling components are expressed in the stimulated cell. Activation of protein kinase A following increased levels of cAMP, for instance, may lead to the phosphorylation (and hence activation) of a number of distinct target proteins, depending on the cell type. Moreover, a given GPCR can link to more than one kind of G protein, and this may depend on the cell type in which the receptor is expressed, or on the nature of the stimulus (see below).

GPCRs are generally considered to be chemical receptors; they sense a wide range of distinct chemicals, from peptides and lipids to nucleotides and ions. However, GPCRs also engage in mechano- and voltage-sensing. It is currently thought that voltage changes may influence certain GPCRs following their stimulation by their ligands. Muscarinic $\mathrm{M}_{2}$ and adrenergic $\alpha_{2} \mathrm{AR}$ receptors are both examples of voltage-sensitive GPCRs, and changes in membrane potential influence signalling events down-stream of GPCR activation in response to activation by their chemical ligand. Hence, cells can respond to their microenvironment in a more facetted way, as the signalling cascaded employed reflects the presence of one or more stimuli.

In contrast, it appears that mechanical stimuli may be the primary stimulus causing activation of other GPCRs. One example of a stretch-activated GPCR is the Angiotensin receptor $\mathrm{AT}_{2} \mathrm{R}$, which can be activated by membrane stretch in the absence of its ligand angiotensin.

GPCRs are generally selective for their agonist but some can be quite promiscuous in terms of ligands that they bind. This is true for a number of GPCRs involved in olfaction, where it is essential to permit the detection of huge range of distinct odorants by a limited number of receptors, as it would be impossible to express selective GPCRs for all possible odorants. Another prominent example is the extracellular $\mathrm{Ca}^{2+}$ sensing receptor, which is involved in body $\mathrm{Ca}^{2+}$ homeostasis. Its main ligand is extracellular $\mathrm{Ca}^{2+}$, but it can also bind $\mathrm{Mg}^{2+}$, polyamines such as spermine, and aromatic amino acids. Intriguingly, the nature of a stimulus or ligand determines which signalling 
cascade is employed, a phenomenon called biased agonism. Hence, even within the same cell and using the same GPCR, distinct signalling cascades can be activated in response to different (combinations of) stimuli.

Since one stimulated GPCR can continue to activate G proteins as long as its ligand is bound, and one $G$ protein-activated enzyme can catalyse many reactions, GPCR activation can cause a huge amplification of the original signal, one ligand binding to one GPCR. Furthermore, once the G protein has been activated, the signalling cascade becomes independent of the presence of the original ligand and is not affected by deactivation of the receptor. Hence, GPCR activation can trigger lengthy signalling cascades and may influence gene transcription, and GPCR actions are often thought of as slow and long lasting. However, G protein subunits can directly interact with ion channels immediately following their dissociation from the activated receptor protein to modify ion channel activity and therefore have the potential to take effect within ms of receptor activation, similar to ion channels.

\section{Carriers}

Another important group of membrane proteins are the carrier proteins. These membrane proteins are not receptors in the classical sense of the term, but their activity does depend on the presence of the chemicals that they transport and they might therefore be considered as sensors of chemical concentrations. Carriers can be divided into transporters and pumps. Their activity generally requires energy expenditure either directly (pumps; in-built ATPase activity; primary active transport) or indirectly (transporters; energy-dependent build-up of gradients required to power transport event; secondary active transport), because - and this is a key difference to channel proteins - transport events can (but don't have to) go against (electro)chemical gradients. Transporters are essential for cell survival as they ensure transport of glucose and other chemicals including amino acids and ions across the membrane, and enable cells to control their volume. Furthermore, without pumps, electric gradients would not be built up, maintained and restored. Testament to the importance of these proteins is the fact that in neurons, the $\mathrm{Na}^{+} / \mathrm{K}^{+}$pump accounts for up to $2 / 3$ of the total energy expenditure.

Transporter activity is determined by the availability of substrate, with transport rates going up with increasing substrate concentrations until saturation 
is reached. Pumps also require ATP levels to be sufficiently high to function, and both types of carrier proteins, like all proteins, are subject to modulation by environmental factors (temperature, $\mathrm{pH}$, electric field, presence or absence of allosteric modulators) and enzyme activity (e.g. phosphorylation).

\section{Relevance to Surgeons}

A thorough understanding of cell physiology is paramount to understanding clinical problems. Aberrant functioning of a very small group of cells, or indeed just one cell, may set into motion a domino effect that can lead to tissue and organ malfunction and give rise to serious pathologies including cancers and degenerative diseases. Appreciation of cell physiology principles furthermore may help anticipate and/or treat complications that may arise during or following surgical procedures. This review emphasised a role for membrane proteins because they play a key role in cell physiology by connecting the intracellular milieu with extracellular events. The importance of membrane receptors is underlined by the fact that GPCRs and ion channels together make up around $60 \%$ of primary targets for clinically prescribed drugs, whilst of the 20,000 - 25,000 genes of the human genome, only around 5\% code for either ion channel proteins (up to 400 genes) or GPCRs (around 800 genes). Hence, a good working understanding of cell physiology helps surgeons understand, predict and improve clinical outcome and treatment strategies. 


\section{Text Box}

\begin{tabular}{|c|c|}
\hline Nusleus & tysosomes \\
\hline 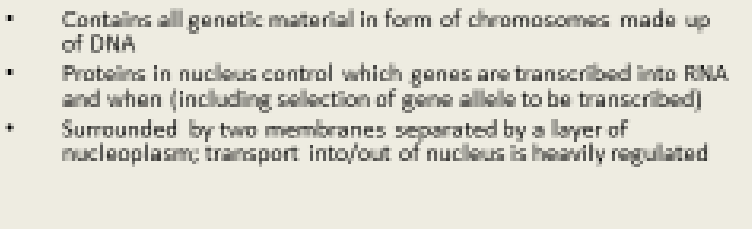 & 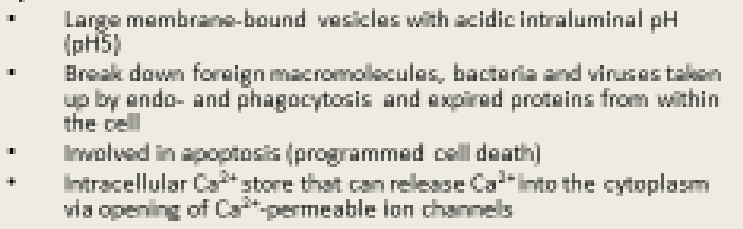 \\
\hline \multicolumn{2}{|r|}{ 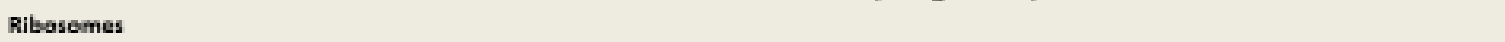 } \\
\hline $\begin{array}{l}\text { - Consists of large and small subunit composed of ribosomal RNA } \\
\text { and protein } \\
\text { + Free in cytosol or bound to ER } \\
\text { - Also found in mitochondria (resemble bacterial ribosomes) }\end{array}$ & $\begin{array}{l}\text { Mitochendria } \\
\text { * Surrounded by two mombranes } \\
\text { * Contain their own circular DNA and (small) ribosomes ("cells } \\
\text { within cells") } \\
\text { * Only passed on from mother to child } \\
\text { * Oxidstive phosphorylation, in which glucose is turned into } \mathrm{CO}_{2} \\
\text { and ATP, the generic energy unit utilised by all living organisms }\end{array}$ \\
\hline $\begin{array}{l}\text { + Made up of stacks of membrane-bound vesicles and sheets that } \\
\text { extend out from the nuclsus (is in part continuousfin contact } \\
\text { with outer membrane of nucleus) into the cytoplasm }\end{array}$ & $\begin{array}{l}\text { * Trigger apoptosis [programmed cell desth) } \\
\text { * } \quad \text { Synthesis of steroids (in cells that produce them) } \\
\text { * } \quad \text { Lipid synthesis and modification }\end{array}$ \\
\hline $\begin{array}{l}\text { * Rough ER has ribosomes attached and is site of protein symthesis; } \\
\text { - Main lipid biosynthesis organelle } \\
\text { + } \quad \text { sends proteins and lipids on to Golgi apparatus for further } \\
\text { modifications }\end{array}$ & $\begin{array}{l}\text { - Heat generation (body thesmoregulation in mammals] } \\
\text { Intracellular } \mathrm{Ca}^{2+} \text { store that can relasese Ca }{ }^{2+} \text { into the cytoplasm } \\
\text { via opening of } \mathrm{Ca}^{2+} \text {-permeable ion chamels }\end{array}$ \\
\hline \multirow[t]{2}{*}{$\begin{array}{l}\text { - Intracellular } \mathrm{Ca}^{2+} \text { store that can reloase } \mathrm{Ca}^{2+} \text { into the cytoplasm } \\
\text { via opening of } \mathrm{Ca}^{2+} \text { permeable ion channels }\end{array}$} & Peroxysomes \\
\hline & $\begin{array}{l}\text { - Membrane-bound vesicles that break down moleculos using } \mathrm{O}_{2} \text {, } \\
\text { including B-oxidstion of fatty acids }\end{array}$ \\
\hline $\begin{array}{l}\text { Golei apparatus } \\
\text { - Made up of series of membrane sacks called cisternae } \\
\text { + Recelves proteins, lipids and some carbohydrates and modifies } \\
\text { them to enable correct trafficking and targeting }\end{array}$ & $\begin{array}{l}\text { - Produce some phosphollpids and cholesterol, as well as bile acids } \\
\text { in liver cells }\end{array}$ \\
\hline & Oytoskeleten \\
\hline & $\begin{array}{l}\text { - Complex network of interacting microfilaments and-tubules } \\
\text { - Provides cell shape, mechanical resistance to deformation, } \\
\text { tethering site for proteins, transport route, chromosome } \\
\text { anchoring site during cell division }\end{array}$ \\
\hline
\end{tabular}

\section{Further reading:}

W. F. Boron, E. L. Boulpaep

\section{Medical Physiology}

( $2^{\text {nd }}$ edn)Elsevier Saunders, Philadelphia (2012)

\section{J. E. Hall}

\section{Textbook of Medical Physiology}

(12 ${ }^{\text {th }}$ edn) Elsevier Saunders, Philadelphia (2011) 